\title{
Direct, indirect and total effects of 13-valent pneumococcal conjugate vaccination on invasive pneumococcal disease in children in Navarra, Spain, 2001 to 2014: cohort and case-control study
} \author{
Working Group for Surveillance of the Pneumococcal Invasive Disease in Navarra 7 \\ 1. Instituto de Salud Pública de Navarra, Pamplona, Spain \\ 2. Navarra Institute for Health Research (IdiSNA), Pamplona, Spain \\ 3. CIBER Epidemiología y Salud Pública (CIBERESP), Spain \\ 4. Department of Clinical Microbiology, Complejo Hospitalario de Navarra, Pamplona, Spain \\ 5. Department of Pediatrics, Complejo Hospitalario de Navarra, Pamplona, Spain \\ 6. Department of Pediatrics, Hospital García Orcoyen, Estella, Spain \\ 7. Members of the group are listed at the end of the article
}

M Guevara ${ }^{123}$, A Barricarte ${ }^{123}$, L Torroba ${ }^{24}$, M Herranz ${ }^{25}$, A Gil-Setas ${ }^{24}$, F Gil 6 , E Bernaola ${ }^{25}$, C Ezpeleta ${ }^{24}$, J Castilla ${ }^{123}$

Correspondence: Marcela Guevara (mguevare@navarra.es)

Guevara M, Barricarte A, Torroba L, Herranz M, Gil-Setas A, Gil F, Bernaola E, Ezpeleta C, Castilla J, Working Group for Surveillance of the Pneumococcal Invasive Disease in Navarra. Direct, indirect and total effects of 13 -valent pneumococcal conjugate vaccination on invasive pneumococcal disease in children in Navarra, Spain, 2001 to 2014: cohort and case-control study. Euro Surveill. 2016;21(14):pii=30186. DOI: http://dx.doi.org/10.2807/1560-7917.ES.2016.21.14.30186

We estimated the direct, indirect and total effects of the 13-valent pneumococcal conjugate vaccine ( $\left.P C V_{13}\right)$ on invasive pneumococcal disease (IPD) in children. A population-based cohort study followed children aged between 2.5 and 59 months between 2001 and 2014 in Navarra, Spain. IPD incidence was compared by PCV status and period. All cases diagnosed from July 2010 to December 2014 and eight matched controls per case were analysed to estimate the adjusted direct effect of PCV13. A total of 120,980 children were followed and 206 IPD cases were detected. Compared with unvaccinated children in the baseline period (2001-2004), overall IPD incidence in $2011-2014$ (76\% average PCV coverage) declined equally in vaccinated (total effect: 76\%; hazard ratio (HR): $0.24 ; 95 \%$ confidence interval (CI): $0.14-0.40$ ) and unvaccinated children (indirect effect: $78 \%$; HR: 0.22 ; $95 \% \mathrm{Cl}: 0.09-0.55$ ). IPD incidence from non-PCV13 serotypes increased among vaccinated children (HR: $2.84 ; 95 \% \mathrm{Cl}: \mathbf{1 . 0 2 - 7 . 8 8}$ ). The direct effect of one or more doses of PCV13 against vaccine serotypes was $95 \%$ (odds ratio: $0.05 ; 95 \% \mathrm{Cl}$ : $0.01-0.55)$. PCV13 was highly effective in preventing vaccine-serotype IPD. The results suggest substantial and similar population-level vaccine benefits in vaccinated and unvaccinated children through strong total and indirect effects.

\section{Introduction}

The 7-valent pneumococcal conjugate vaccine $\left(\mathrm{PCV}_{7}\right)$ has proved highly effective in preventing invasive pneumococcal disease (IPD) caused by the serotypes included in its formulation $[1,2]$. However, its impact has varied across countries due to factors that may include differences in serotype distribution, vaccination coverage and characteristics of vaccination programmes [3,4]. New, higher valency pneumococcal conjugate vaccines (PCVs) containing 10 (PCV10) and 13 ( $\left.P \mathrm{CV}_{13}\right)$ serotypes were licensed on the basis of non-inferiority of immunogenicity compared with $\mathrm{PCV}_{7}$ [5]; thus, post-licensure studies are required to assess their effects under real-life conditions.

To date (June 2015), there are few studies published on the direct effect of $\mathrm{PCV}_{13}$ against IPD, and all except one have used the indirect cohort method and have hence been limited to evaluating it against vaccine serotypes rather than total IPD [6-8]. PCVs can also reduce IPD incidence among unvaccinated individuals as a result of reduced transmission. This indirect or 'herd' effect has been studied in unvaccinated age groups [9-12], but not in children targeted for vaccination. The total effect accounts for both the direct and indirect effects on vaccinated individuals $[13,14]$.

In Navarra, Spain, PCVs became available for private purchase in June 2001 (PCV7), November 2009 (PCV10) and June 2010 (PCV13), and are publicly funded only for children with selected IPD risk factors, including cardiovascular, respiratory, neurological, renal or hepatic disease, diabetes, cancer, immunosuppression, HIV infection, haemoglobinopathy, and cerebrospinal fluid leak [15]. The Spanish Association of Paediatrics recommends PCV for all children younger than 5 years [16], and coverage has increased progressively through 


\section{FIGURE}

Scheme of the main study groups and the comparisons made, cohort and case-control study, effects of pneumococcal conjugate vaccination on invasive pneumococcal disease in children, Navarra, Spain, 2001-2014

\section{Cohort study}

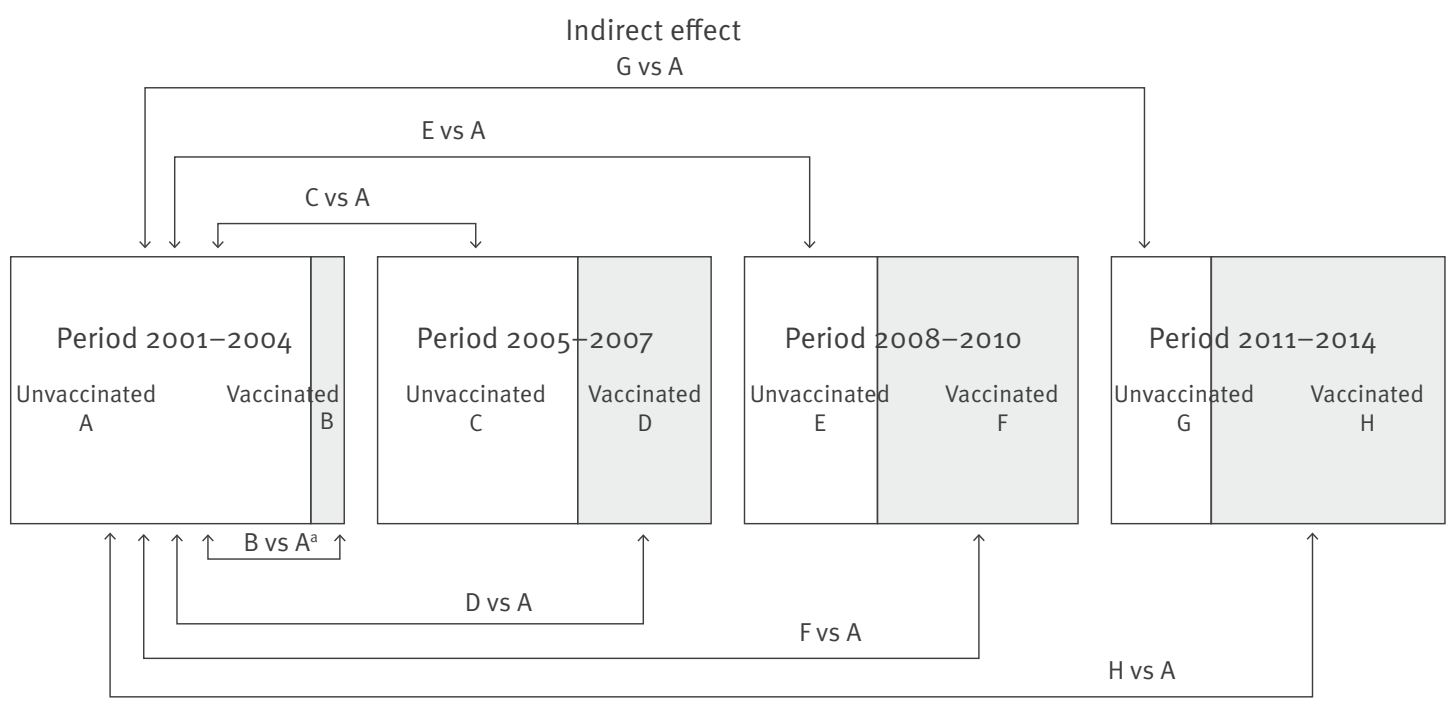

Total effect

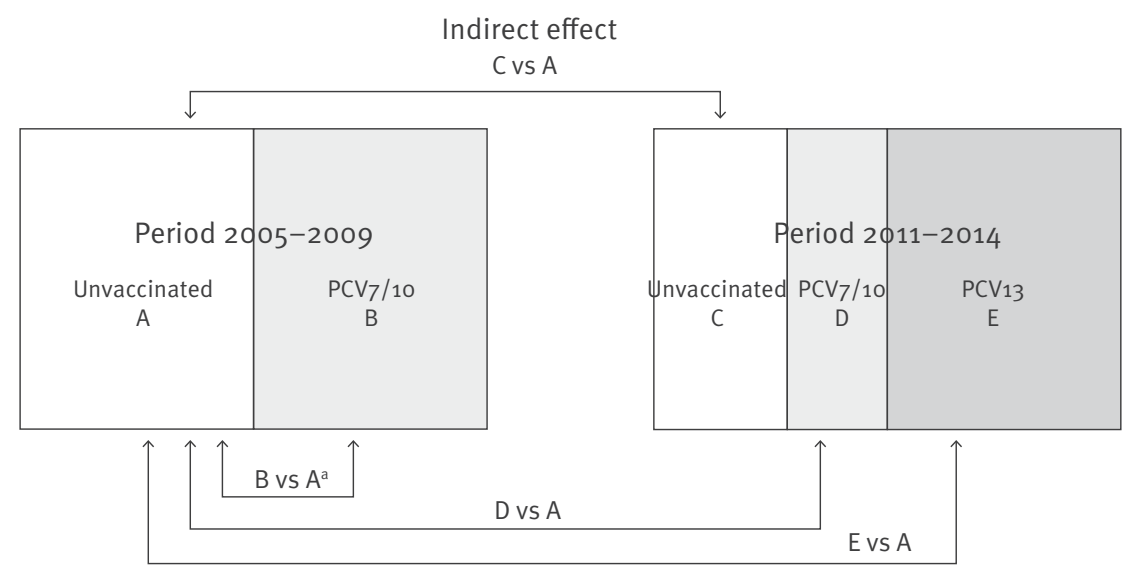

Total effect

\section{Case-control study}

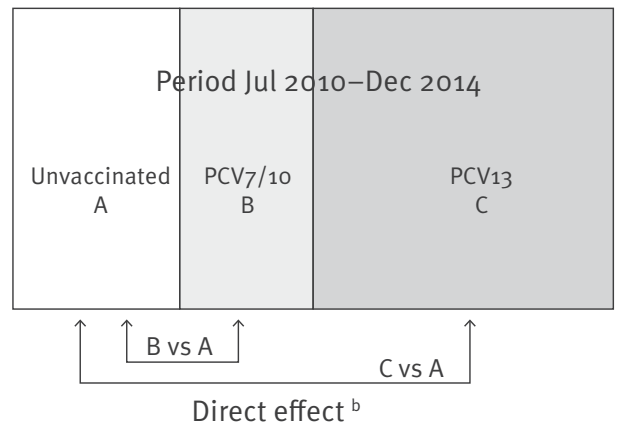

PCV: pneumococcal conjugate vaccine.

a B vs $A$ in the cohort study is the direct effect in the baseline period.

b The direct effect in the case-control study is estimated by the odds ratio of vaccination vs non-vaccination in cases compared with controls. 
Incidence of invasive pneumococcal disease in children younger than 5 years and younger than 2 years by conjugate pneumococcal vaccine status and period, Navarra, Spain, 2001-2014

\begin{tabular}{|c|c|c|c|c|c|c|c|c|c|c|}
\hline \multirow[b]{2}{*}{ Age } & \multirow[b]{2}{*}{ Period } & \multicolumn{3}{|c|}{ Unvaccinated with PCV } & \multicolumn{3}{|c|}{ Vaccinated with PCV } & \multicolumn{3}{|c|}{ Total } \\
\hline & & $\begin{array}{l}\text { Number } \\
\text { of cases }\end{array}$ & PY & $\begin{array}{c}\text { Incidence rate } \\
\text { per } 100,000 \\
\text { PY }\end{array}$ & $\begin{array}{l}\text { Number } \\
\text { of cases }\end{array}$ & PY & $\begin{array}{l}\text { Incidence rate } \\
\text { per } 100,000 \text { PY }\end{array}$ & $\begin{array}{l}\text { Number } \\
\text { of cases }\end{array}$ & PY & $\begin{array}{l}\text { Incidence } \\
\text { rate per } \\
100,000 \mathrm{PY}\end{array}$ \\
\hline \multirow{4}{*}{$\begin{array}{l}75 \text { days } \\
\text { to } 59 \\
\text { months }\end{array}$} & 2001-2004 & 77 & 102,845 & 75 & 10 & 13,162 & 76 & 87 & 116,007 & 75 \\
\hline & $2005-2007$ & 27 & 47,597 & 57 & 33 & 43,232 & 76 & 60 & 90,829 & 66 \\
\hline & $2008-2010$ & 13 & 32,025 & 41 & 23 & 61,310 & 38 & 36 & 93,335 & 39 \\
\hline & $2011-2014$ & 5 & 30,482 & 16 & 18 & 94,454 & 19 & 23 & 124,937 & 18 \\
\hline \multirow{4}{*}{$\begin{array}{l}75 \text { days } \\
\text { to } 23 \\
\text { months }\end{array}$} & 2001-2004 & 51 & 34,626 & 147 & 9 & 7,014 & 128 & 60 & 41,640 & 144 \\
\hline & $2005-2007$ & 18 & 14,331 & 126 & 18 & 17,805 & 101 & 36 & 32,136 & 112 \\
\hline & $2008-2010$ & 8 & 10,700 & 75 & 11 & 22,697 & 48 & 19 & 33,397 & 57 \\
\hline & $2011-2014$ & 3 & 9,872 & 30 & 13 & 34,731 & 37 & 16 & 44,603 & 36 \\
\hline
\end{tabular}

PCV: pneumococcal conjugate vaccine; PY: person-years.

Culture-negative cases were excluded ( $n=0,2,6$ and 5 in each period, respectively).

the private market, reaching $78 \%$ in children up to 23 months of age in 2013 [17]. Most of the vaccinated children have received a complete $3+1$ schedule, with doses at 2, 4 and 6 months plus a booster dose at 12-15 months. Since 2010, PCV13 has been the predominant PCV in use. After the change from $\mathrm{PCV}_{7}$ to $P C V_{13}, I P D$ incidence from all serotypes decreased by $69 \%$, from 60.7 to 18.7 cases/100,000 inhabitants, in children younger than 5 years [17], in line with what has been observed in other countries [10,11,18-21].

The aim of this study was to estimate the effect of $P C V_{13}$ on IPD incidence in vaccinated (direct and total effects) and unvaccinated (indirect effect) children younger than 5 years.

\section{Methods}

\section{Study design}

A population-based cohort study with follow-up during 2001-2014, and a nested case-control study from July 2010 to December 2014 were conducted in Navarra, a region with ca 640,000 inhabitants, including ca 34,700 aged less than 5 years, in 2014 [22]. The Navarra Ethical Committee for Medical Research approved the study protocol.

\section{Sources of information and variables}

The Navarra Health Service provides healthcare, free at point of service, to $97 \%$ of the inhabitants of the region. Clinical records have been computerised since 2000 and include reports from primary care, hospital admissions, the regional vaccination register, and laboratory test results.

Vaccination history was obtained from the regional vaccination register [23], which includes all doses received by children, including those acquired in the private market. Vaccine doses were counted starting 15 days after their administration and the 14 days after receiving the first dose were not considered for the analysis. Cases of IPD were identified through the active laboratory-based surveillance. IPD was defined as isolation, PCR or antigen detection of Streptococcus pneumoniae from a normally sterile body site. Pneumococcal isolates were serotyped at the national reference laboratory (Instituto de Salud Carlos III, Madrid) by the Quellung reaction or dot-blot assay, and were classified as $\mathrm{PCV}_{7}$ serotypes (4, 6B, 9V, 14, 18C, 19F, 23F), additional $P C V_{13}$ serotypes $(1,3,5,6 \mathrm{~A}, 7 \mathrm{~F}, 19 \mathrm{~A})$, or non-PCV13 serotypes.

\section{Cohort study to evaluate the indirect and total vaccine effects}

The cohort included children covered by the Navarra Health Service from birth to their fifth birthday, the end of the follow-up on 31 December 2014 or date of death, whichever occurred first. Cox regression was performed to obtain hazard ratios (HR) with their 95\% confidence intervals $(\mathrm{Cl})$. Age in days was used as the underlying time scale, with entry time defined as age at 1 January 2001 or 75 days of age if it was later, and exit time as 59 months of age, age at IPD diagnosis or death, or at 31 December 2014, whichever occurred first. Calendar periods and PCV status were defined as time-dependent variables. Person-years (PY) at risk were used as the denominators of the IPD incidence rates. Since a culture was taken from all suspected cases and PCR and antigen detection were progressively introduced as complementary tests, culturenegative cases $(n=13)$ were excluded to maintain comparability between the periods.

To evaluate the indirect and total effects of the PCVs with respect to the pre-vaccine situation, we considered four periods according to PCV use and coverage in children younger than 5 years: the baseline period (2001-2004) during which PCV7 use was low; the 
Estimates of the indirect and total effects of the pneumococcal conjugate vaccines, with unvaccinated children younger than 5 years in the period with low use of pneumococcal conjugate vaccine (2001-2004) as reference category, Navarra, Spain, 2001-2014

\begin{tabular}{|c|c|c|c|c|c|c|c|}
\hline \multirow[b]{2}{*}{ Serotypes } & \multirow[b]{2}{*}{ Period } & \multicolumn{3}{|c|}{ Unvaccinated with PCV (indirect effect) ${ }^{a}$} & \multicolumn{3}{|c|}{ Vaccinated with PCV (total effect) ${ }^{a}$} \\
\hline & & Number of cases & $\begin{array}{l}\text { Hazard ratio } \\
(95 \% \mathrm{Cl})\end{array}$ & $p$ value & Number of cases & $\begin{array}{c}\text { Hazard ratio } \\
(95 \% \mathrm{Cl})\end{array}$ & $\mathrm{p}$ value \\
\hline \multirow{4}{*}{ All serotypes } & $2001-2004$ & 77 & \multicolumn{2}{|c|}{ Reference } & 10 & $\begin{array}{c}0.77 \\
(0.40-1.49) \\
\end{array}$ & 0.430 \\
\hline & $2005-2007$ & 27 & $\begin{array}{c}0.80 \\
(0.52-1.30) \\
\end{array}$ & 0.317 & 33 & $\begin{array}{c}0.90 \\
(0.60-1.35) \\
\end{array}$ & 0.589 \\
\hline & $2008-2010$ & 13 & $\begin{array}{c}0.55 \\
(0.30-0.98) \\
\end{array}$ & 0.044 & 23 & $\begin{array}{c}0.47 \\
(0.29-0.74) \\
\end{array}$ & 0.001 \\
\hline & $2011-2014$ & 5 & $\begin{array}{c}0.22 \\
(0.09-0.55) \\
\end{array}$ & 0.001 & 18 & $\begin{array}{c}0.24 \\
(0.14-0.40) \\
\end{array}$ & $<0.001$ \\
\hline \multirow{4}{*}{$\mathrm{PCV}_{7}$ serotypes $^{\mathrm{b}}$} & $2001-2004$ & 36 & \multicolumn{2}{|c|}{ Reference } & 1 & $0.15(0.02-1.08)$ & 0.058 \\
\hline & $2005-2007$ & 4 & $\begin{array}{c}0.26 \\
(0.09-0.73)\end{array}$ & 0.011 & 0 & 0 (o.oo-UD) & $<0.001^{c}$ \\
\hline & $2008-2010$ & 1 & $\begin{array}{c}0.09 \\
(0.01-0.66) \\
\end{array}$ & 0.018 & 0 & $0(0.00-U D)$ & $<0.001^{c}$ \\
\hline & $2011-2014$ & 1 & $\begin{array}{c}0.10 \\
(0.01-0.70)\end{array}$ & 0.021 & 0 & $0(0.00-U D)$ & $<0.001^{c}$ \\
\hline \multirow{4}{*}{$\begin{array}{l}\text { Additional PCV13 } \\
\text { serotypes }^{\text {b }}\end{array}$} & 2001-2004 & 34 & \multicolumn{2}{|c|}{ Reference } & 6 & $1.08\left(0.45^{-2.57)}\right.$ & 0.872 \\
\hline & $2005-2007$ & 15 & $\begin{array}{c}1.00 \\
(0.54-1.83) \\
\end{array}$ & 0.987 & 22 & $1.37(0.80-2.35)$ & 0.251 \\
\hline & $2008-2010$ & 9 & $\begin{array}{c}0.86 \\
(0.41-1.79) \\
\end{array}$ & 0.679 & 17 & $0.79(0.44-1.41)$ & 0.418 \\
\hline & $2011-2014$ & 4 & $\begin{array}{c}0.40 \\
(0.14-1.14) \\
\end{array}$ & 0.085 & 3 & $\begin{array}{c}0.09 \\
(0.03-0.30) \\
\end{array}$ & $<0.001$ \\
\hline \multirow{4}{*}{ Non-PCV13 serotypes ${ }^{b}$} & 2001-2004 & 5 & \multicolumn{2}{|c|}{ Reference } & 3 & $\begin{array}{c}3.43 \\
(0.82-14.42) \\
\end{array}$ & 0.092 \\
\hline & $2005-2007$ & 4 & $\begin{array}{c}1.85 \\
(0.50-6.90) \\
\end{array}$ & 0.358 & 9 & $\begin{array}{c}3.66 \\
(1.23-10.94) \\
\end{array}$ & 0.020 \\
\hline & $2008-2010$ & 3 & $\begin{array}{c}1.94 \\
(0.46-8.10) \\
\end{array}$ & 0.366 & 6 & $\begin{array}{c}1.84 \\
(0.56-6.04) \\
\end{array}$ & 0.313 \\
\hline & $2011-2014$ & 0 & $0(0.00-U D)$ & $0.273^{c}$ & 14 & $2.84(1.02-7.88)$ & 0.045 \\
\hline
\end{tabular}

$\mathrm{Cl}$ : confidence interval; PCV: pneumococcal conjugate vaccine; UD: undefined.

a Cox regression adjusted for age as underlying time scale, sex and a variable that combines time periods and vaccination status.

${ }^{b}$ Non-typed cases were excluded from analyses by serotype group $(n=2,6,0$ and 1 in each period, respectively).

c $p$ value obtained by the two-tailed mid-p exact test without specific correction.

period of increased PCV7 coverage (2005-2007); the period of high $\mathrm{PCV}_{7}$ coverage and transition to higher valency PCVs (2008-2010); and the period of PCV13 use (2011-2014). Children were considered as vaccinated if they had received at least one dose of PCV. The cohort analysis used a variable that combines time periods and vaccination status, while also adjusting for sex.

In another cohort analysis we evaluated the specific effect of the change from $\mathrm{PCV}_{7}$ to $\mathrm{PCV}_{13}$, considering two periods: $2005-2009$, or the period of $\mathrm{PCV}_{7}$ use; and 2011-2014, or the period of PCV13 use. Three exclusive categories of vaccination status were defined in the following order: at least one dose of $\mathrm{PCV}_{13}$, at least one dose of $\mathrm{PCV}_{7}$ or $P C V_{10}$, and no dose of PCV. The year 2010 was excluded from this analysis because this was a transition year with appreciable use of $\mathrm{PCV}_{7}$, PCV10 and PCV13.
The incidence of IPD in unvaccinated children during the baseline period was used as the reference to estimate the indirect effect by comparison with the IPD incidence in unvaccinated children in each PCV period, and to estimate the total effect by comparison with the incidence in vaccinated children in each PCV period (Figure) $[13,14]$. Where zero cases were observed in one group, the $p$ value was obtained by the two-tailed mid-p exact test.

\section{Case-control study to evaluate the direct vaccine effect}

A case-control study, nested within the cohort, included as case patients all children born since June 2008 (as they might have received at least one dose of $\left.P C V_{13}\right)$ and who were diagnosed with IPD by culture, PCR or antigen detection between July 2010 and December 2014. For each case, eight controls were selected from children with no previous IPD, individually matched by 
Estimates of the indirect and total effects of the pneumococcal conjugate vaccines, with unvaccinated children in the period 2005-2009 (period of use of 7-valent pneumococcal conjugate vaccine) as reference category, Navarra, Spain, 2005-2014

\begin{tabular}{|c|c|c|c|c|}
\hline & Number of cases & Person-years & Hazard ratio $(95 \% \mathrm{Cl})^{\text {a }}$ & $\mathrm{p}$ value \\
\hline \multicolumn{5}{|l|}{ All serotypes } \\
\hline Period 2005-2009, unvaccinated & 37 & 69,667 & \multicolumn{2}{|l|}{ Reference } \\
\hline Period 2005-2009, vaccinated with $P C V_{7 / 10}$ & 52 & 82,667 & $1.03(0.67-1.58)$ & 0.889 \\
\hline Period 2011-2014, unvaccinated (indirect effect) & 5 & 30,482 & $0.30(0.12-0.77)$ & 0.012 \\
\hline Period 2011-2014, vaccinated with PCV7/10 (total effect) & 1 & 29,976 & $0.09(0.01-0.65)$ & 0.017 \\
\hline Period 2011-2014, vaccinated with PCV13 (total effect) & 17 & 64,376 & $0.39(0.22-0.70)$ & 0.002 \\
\hline \multicolumn{5}{|l|}{$\mathrm{PCV}_{13}$ serotypes $^{\mathrm{b}}$} \\
\hline Period 2005-2009, unvaccinated & 27 & 69,667 & \multicolumn{2}{|l|}{ Reference } \\
\hline Period 2005-2009, vaccinated with PCV $7 / 10$ & 36 & 82,667 & $0.92(0.58-1.46)$ & 0.714 \\
\hline Period 2011-2014, unvaccinated (indirect effect) & 5 & 30,482 & $0.42(0.16-1.08)$ & 0.071 \\
\hline Period 2011-2014, vaccinated with $\mathrm{PCV}_{7} / 10$ (total effect) & o & 29,976 & 0 (o.0o-UD) & $<0.001^{\mathrm{c}}$ \\
\hline Period 2011-2014, vaccinated with PCV13 (total effect) & 3 & 64,376 & $0.10(0.03-0.32)$ & $<0.001$ \\
\hline \multicolumn{5}{|l|}{ Non-PCV13 serotypes ${ }^{b}$} \\
\hline Period 2005-2009, unvaccinated & 6 & 69,667 & \multicolumn{2}{|l|}{ Reference } \\
\hline Period 2005-2009, vaccinated with $\mathrm{PCV} 7 / 10$ & 14 & 82,667 & $1.63(0.62-4.24)$ & 0.321 \\
\hline Period 2011-2014, unvaccinated (indirect effect) & 0 & 30,482 & 0 (o.00-UD) & $0.113^{c}$ \\
\hline Period 2011-2014, vaccinated with PCV7/10 (total effect) & 1 & 29,976 & $0.69(0.08-5.94)$ & 0.737 \\
\hline Period 2011-2014, vaccinated with PCV13 (total effect) & 13 & 64,376 & $1.67(0.63-4.43)$ & 0.305 \\
\hline
\end{tabular}

$\mathrm{Cl}$ : confidence interval; NA: not applicable; PCV: pneumococcal conjugate vaccine; UD: undefined.

${ }^{a}$ Cox regression adjusted for age as underlying time scale, sex and a variable that combines time periods and vaccination status.

${ }^{b}$ Non-typed cases were excluded from analyses by serotype group ( $n=6$ and 1 in each period, respectively).

c $p$ value obtained by the two-tailed mid-p exact test without specific correction.

paediatric practice, district of residence and date of birth ( \pm 2 months). Of all the children who met these eligibility criteria, the eight with dates of birth closest to that of the case were selected. Previous inclusion of a twin was an exclusion criterion.

Healthcare computerised databases were used to obtain the sex, date of birth, paediatrician, district of residence, premature birth ('37 weeks’ gestation), low birth weight $(<2,500 \mathrm{~g})$, major chronic illness (defined as cardiovascular, respiratory, neurological, renal or hepatic disease, diabetes, immunosuppression or cancer), primary care visits in the previous 12 months, other children younger than 5 years in the household, and parental income level ('EUR 18,000 and $\geq$ EUR 18,000/year).

The reference date for cases was the date of symptom onset, and for controls, the date on which their age exactly matched the age in days of their corresponding case at the time of symptom onset. Different categorisations of PCV status were used to analyse the effect of either PCV13 including mixed schedules or PCV13-only schedules, with or without distinction of the number of doses received. Vaccination with $\mathrm{PCV}_{7}$ or $\mathrm{PCV}_{10}$ without $P C V_{13}$, and non-PCV vaccination were assigned to two separate categories.
In different analyses we evaluated the effect of receiving PCV13 on the risk of IPD due to all serotypes, to $P C V_{13}$ serotypes, to additional $P C_{13}$ serotypes and to non-PCV13 serotypes, using non-PCV vaccination as the reference. A sensitivity analysis was performed excluding children with any medical condition. Adjusted matched odds ratios (OR), with their $95 \% \mathrm{Cl}$, were calculated using conditional logistic regression. We assessed for confounding by including additional variables one by one in the model. Covariates were removed if they did not change the OR by at least $15 \%$. Vaccine effects were calculated as $(1-H R) \times 100$ or $(1-O R) \times 100$. Two-tailed $p$ values $<0.05$ were considered to be statistically significant.

\section{Results}

\section{Evaluation of the indirect and total vaccine effects}

Between 2001 and 2014, 120,980 children were followed. In the periods 2001-2004, 2005-2007, 20082010 and 2011-2014, we registered 116,007 PY, 90,829 PY, 93,335 PY and 124,937 PY of follow-up, of which $11 \%, 48 \%, 66 \%$ and $76 \%$, respectively, corresponded to children who had received at least one dose of PCV. Considering the PCV with the highest valency received, in the period $2011-2014,52 \%$ of PY corresponded to children with at least one dose of PCV13, 6\% of PCV 10 and $18 \%$ of PCV7. During the follow-up, 206 cases of 
Characteristics of cases and controls included in the case-control study of the direct effect of pneumococcal conjugate vaccination on invasive pneumococcal disease in children, Navarra, Spain, July 2010-December 2014

\begin{tabular}{|c|c|c|c|}
\hline & $\begin{array}{l}\text { Cases } \\
\mathrm{n}=34\end{array}$ & $\begin{array}{c}\text { Controls } \\
n=272\end{array}$ & $\begin{array}{c}p \text { value } \\
\text { (matched) }\end{array}$ \\
\hline \multicolumn{4}{|l|}{ Demographics } \\
\hline Median age in months (range) & $18.9(3.4-57.7)$ & $18.9(3.4-57.7)$ & NA \\
\hline Male sex & $24(71 \%)$ & $139(51 \%)$ & 0.037 \\
\hline Resides in urban area & $23(68 \%)$ & $184(68 \%)$ & 1.000 \\
\hline \multicolumn{4}{|l|}{ Number of other persons in household } \\
\hline $1-2$ & $7(21 \%)$ & $73(27 \%)$ & \multirow{3}{*}{0.476} \\
\hline 3 & $12(35 \%)$ & $107(39 \%)$ & \\
\hline$\geq 4$ & $15(44 \%)$ & $92(34 \%)$ & \\
\hline Other children younger than 5 years in household & $16(47 \%)$ & $102(38 \%)$ & 0.282 \\
\hline \multicolumn{4}{|l|}{ Parental income level } \\
\hline 〈EUR 18,00o/year & $19(56 \%)$ & $148(54 \%)$ & \multirow{2}{*}{0.868} \\
\hline$\geq$ EUR 18,00o/year & $15(44 \%)$ & $124(46 \%)$ & \\
\hline \multicolumn{4}{|l|}{ Primary care visits in the previous year } \\
\hline $0-2$ & $6(18 \%)$ & $58(21 \%)$ & \multirow{3}{*}{0.309} \\
\hline $3-7$ & $17(50 \%)$ & $100(37 \%)$ & \\
\hline$\geq 8$ & $11(32 \%)$ & $114(42 \%)$ & \\
\hline \multicolumn{4}{|l|}{ Medical conditions } \\
\hline Major chronic illness a & $1(3 \%)$ & $7(3 \%)$ & 0.899 \\
\hline Premature birth or low birth weight & $2(6 \%)$ & $11(4 \%)$ & 0.626 \\
\hline Any underlying medical condition ${ }^{\mathrm{b}}$ & $3(9 \%)$ & $15(6 \%)$ & 0.451 \\
\hline \multicolumn{4}{|l|}{ Vaccination history } \\
\hline Meningococcal C conjugate vaccine & $34(100 \%)$ & $272(100 \%)$ & 1.000 \\
\hline 23-valent pneumococcal polysaccharide vaccine & $0(0 \%)$ & $\mathrm{o}(\mathrm{o} \%)$ & 1.000 \\
\hline At least one dose of any PCV & $27(79 \%)$ & $217(80 \%)$ & 0.957 \\
\hline At least three doses of any PCV & $19(56 \%)$ & $164(60 \%)$ & 0.552 \\
\hline \multicolumn{4}{|l|}{ Highest valency PCV received } \\
\hline No $P C V$ vaccination & $7(21 \%)$ & $55(20 \%)$ & \multirow{4}{*}{0.955} \\
\hline $\mathrm{PCV}_{7}$ & $2(6 \%)$ & $12(4 \%)$ & \\
\hline PCV10 & $1(3 \%)$ & $7(3 \%)$ & \\
\hline $\mathrm{PCV}_{13}$ & $24(71 \%)$ & $198(73 \%)$ & \\
\hline
\end{tabular}

PCV: pneumococcal conjugate vaccine; NA: not applicable.

${ }^{a}$ Defined as cardiovascular, respiratory, neurological, renal or hepatic disease, diabetes, immunosuppression or cancer.

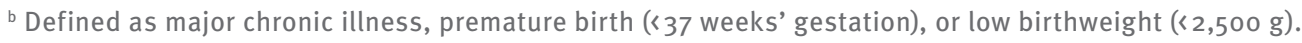

IPD were registered, 84 of them in children who had received at least one dose of $P C V$. The IPD incidence rates decreased over the four periods: from 75 to 66 , 39 and 18 per 100,000 PY, respectively (Table 1).

In the reference group, unvaccinated children in the period 2001-2004, 48\% (36/75) of the serotyped cases were due to $\mathrm{PCV}_{7}$ serotypes and $93 \%$ (70/75) to PCV13 serotypes. We observed an increasing indirect effect of the PCVs in preventing IPD from all serotypes in unvaccinated children, which reached 45\% (HR: 0.55; 95\% Cl: $0.30-0.98$ ) in 2008-2010 and 78\% (HR: 0.22 ; 95\% Cl: $0.09-0.55)$ in 2011-2014. This indirect effect was similar to the total (direct and indirect) protective effect in vaccinated children, which reached $53 \%$ (HR:
$0.47 ; 95 \% \mathrm{Cl}: 0.29-0.74)$ in $2008-2010$ and $76 \%$ (HR: $0.24 ; 95 \% \mathrm{Cl}: 0.14-0.40$ ) in 2011-2014 (Table 2).

The PCV effect in preventing IPD due to $\mathrm{PCV}_{7}$ serotypes was earlier and more pronounced in vaccinated than in unvaccinated children, with only one vaccine failure in the period 2001-2004, and 100\% total effect from 2005 to 2014. Unvaccinated children showed an important indirect effect of $74 \%$ ( $\mathrm{HR}: 0.26 ; 95 \% \mathrm{Cl}: 0.09-0.73$ ) in the period 2005-2007 and 90\% (HR: 0.10; 95\% Cl: 0.01-0.70) in 2011-2014. In the latter period the total effect against IPD due to additional PCV13 serotypes was 91\% (HR: 0.09; 95\% Cl: 0.03-0.30). The incidence of IPD due to non-PCV13 serotypes in vaccinated children in the period 2011-2014 was higher than in the 


\section{TABLE 5}

Estimates of the direct effect of the 13-valent pneumococcal conjugate vaccine and other pneumococcal conjugate vaccines in the case-control study, Navarra, Spain, July 2010-December 2014

\begin{tabular}{|c|c|c|c|c|c|c|}
\hline \multirow[b]{2}{*}{ Invasive pneumococcal disease serotypes } & \multirow{2}{*}{$\begin{array}{l}\text { Cases } \\
\text { Vac./ } \\
\text { Unvac. }\end{array}$} & \multirow{2}{*}{$\begin{array}{c}\text { Controls } \\
\text { Vac./ } \\
\text { Unvac. }\end{array}$} & \multicolumn{2}{|c|}{ Crude effect $t^{a}$} & \multicolumn{2}{|c|}{ Adjusted effect ${ }^{\mathrm{b}}$} \\
\hline & & & $\begin{array}{c}\text { OR } \\
(95 \% \mathrm{Cl})\end{array}$ & $\mathrm{p}$ value & $\begin{array}{c}\text { OR } \\
(95 \% \mathrm{Cl})\end{array}$ & $\mathrm{p}$ value \\
\hline \multicolumn{7}{|l|}{ All serotypes } \\
\hline$\geq 1$ dose $P C V_{7} / 10$, no $P C V_{13}$ & $3 / 7$ & $19 / 55$ & $\begin{array}{c}1.35 \\
(0.28-6.42)\end{array}$ & 0.708 & $\begin{array}{c}1.28 \\
(0.25-6.52) \\
\end{array}$ & 0.766 \\
\hline$\geq 1$ dose $P C V_{13}$ (including mix) & $24 / 7$ & $198 / 55$ & $\begin{array}{c}0.90 \\
(0.33-2.41) \\
\end{array}$ & 0.828 & $\begin{array}{c}0.91 \\
(0.33-2.49) \\
\end{array}$ & 0.848 \\
\hline$\geq 1$ dose $P C V_{13}$ only & $21 / 7$ & $174 / 55$ & $\begin{array}{c}0.85 \\
(0.29-2.50) \\
\end{array}$ & 0.770 & $\begin{array}{c}0.86 \\
(0.29-2.56) \\
\end{array}$ & 0.788 \\
\hline$\geq 3$ doses $\mathrm{PCV}_{13}$ & $14 / 7$ & $123 / 55$ & $\begin{array}{c}0.75 \\
(0.24-2.36)\end{array}$ & 0.622 & $\begin{array}{c}0.75 \\
(0.23-2.42)\end{array}$ & 0.629 \\
\hline $\begin{array}{l}\geq 1 \text { dose } P C V_{13} \text {, excluding children with any medical } \\
\text { condition }{ }^{c}\end{array}$ & $21 / 7$ & $186 / 52$ & $\begin{array}{c}0.82 \\
(0.31-2.21)\end{array}$ & 0.703 & $\begin{array}{c}0.82 \\
(0.30-2.24)\end{array}$ & 0.697 \\
\hline \multicolumn{7}{|l|}{ PCV13 serotypes } \\
\hline$\geq 1$ dose $\mathrm{PCV}_{7} / 10$, no $\mathrm{PCV} 13$ & $1 / 6$ & $14 / 19$ & $\begin{array}{c}0.33 \\
(0.03-3.27)\end{array}$ & 0.341 & $\begin{array}{c}0.09 \\
(0.01-1.66)\end{array}$ & 0.106 \\
\hline$\geq 1$ dose $P C_{13}$ (including mix) & $3 / 6$ & $47 / 19$ & $\begin{array}{c}0.15 \\
(0.03-0.85)\end{array}$ & 0.032 & $\begin{array}{c}0.05 \\
(0.01-0.55)\end{array}$ & 0.014 \\
\hline$\geq 1$ dose $\mathrm{PCV}_{13}$ only & $2 / 6$ & $40 / 19$ & $\begin{array}{c}0.08 \\
(0.01-0.76) \\
\end{array}$ & 0.028 & $\begin{array}{c}0.04 \\
(0.00-0.57) \\
\end{array}$ & 0.018 \\
\hline$\geq 3$ doses $P C V_{13}$ & $2 / 6$ & $35 / 19$ & $\begin{array}{c}0.10 \\
(0.01-0.96)\end{array}$ & 0.046 & $\begin{array}{c}0.04 \\
(0.00-0.61)\end{array}$ & 0.021 \\
\hline $\begin{array}{l}\geq 1 \text { dose } \mathrm{PCV}_{13} \text {, excluding children with any medical } \\
\text { condition }^{c}\end{array}$ & $3 / 6$ & $44 / 19$ & $\begin{array}{c}0.16 \\
(0.03-0.90) \\
\end{array}$ & 0.037 & $\begin{array}{c}0.06 \\
(0.01-0.58) \\
\end{array}$ & 0.015 \\
\hline \multicolumn{7}{|l|}{ Additional PCV13 serotypes } \\
\hline$\geq 1$ dose $\mathrm{PCV}_{7} / 10$, no $\mathrm{PCV} 13$ & $1 / 5$ & $11 / 14$ & $\begin{array}{c}0.41 \\
(0.04-4.54)\end{array}$ & 0.466 & $\begin{array}{c}0.13 \\
(0.01-2.45)\end{array}$ & 0.174 \\
\hline$\geq 1$ dose $P_{C} 13$ (including mix) & $3 / 5$ & $47 / 14$ & $\begin{array}{c}0.15 \\
(0.03-0.86) \\
\end{array}$ & 0.033 & $\begin{array}{c}0.07 \\
(0.01-0.70) \\
\end{array}$ & 0.023 \\
\hline$\geq 1$ dose $\mathrm{PCV}_{13}$ only & $2 / 5$ & $40 / 14$ & $\begin{array}{c}0.08 \\
(0.01-0.77)\end{array}$ & 0.029 & $\begin{array}{c}0.05 \\
(0.00-0.70)\end{array}$ & 0.027 \\
\hline$\geq 3$ doses $P_{C} V_{13}$ & $2 / 5$ & $35 / 14$ & $\begin{array}{c}0.10 \\
(0.01-0.97) \\
\end{array}$ & 0.047 & $\begin{array}{c}0.05 \\
(0.00-0.76) \\
\end{array}$ & 0.031 \\
\hline $\begin{array}{l}\geq 1 \text { dose } \mathrm{PCV}_{13} \text {, excluding children with any medical } \\
\text { condition }^{c}\end{array}$ & $3 / 5$ & $44 / 14$ & $\begin{array}{c}0.16 \\
(0.03-0.91) \\
\end{array}$ & 0.039 & $\begin{array}{c}0.08 \\
(0.01-0.73) \\
\end{array}$ & 0.026 \\
\hline \multicolumn{7}{|l|}{ Non-PCV13 serotypes } \\
\hline$\geq 1$ dose $\mathrm{PCV}_{7} / 10$, no $\mathrm{PCV} 13$ & $1 / 1$ & $5 / 31$ & $\begin{array}{c}5.94 \\
(0.32-111.11) \\
\end{array}$ & 0.233 & $\begin{array}{c}7.66 \\
(0.38-154.19) \\
\end{array}$ & 0.184 \\
\hline$\geq 1$ dose $\mathrm{PCV}_{13}$ (including mix) & $18 / 1$ & $124 / 31$ & $\begin{array}{c}5.44 \\
(0.64-46.17) \\
\end{array}$ & 0.121 & $\begin{array}{c}6.75 \\
(0.77-59.19) \\
\end{array}$ & 0.085 \\
\hline$\geq 1$ dose $\mathrm{PCV}_{13}$ only & $16 / 1$ & $114 / 31$ & $\begin{array}{c}5.03 \\
(0.50-50.33) \\
\end{array}$ & 0.169 & $\begin{array}{c}5.70 \\
(0.59-55.23) \\
\end{array}$ & 0.133 \\
\hline$\geq 3$ doses $\mathrm{PCV}_{13}$ & $9 / 1$ & $69 / 31$ & $\begin{array}{c}4.48 \\
(0.40-50.06) \\
\end{array}$ & 0.223 & $\begin{array}{c}5.08 \\
(0.43-59.53) \\
\end{array}$ & 0.196 \\
\hline $\begin{array}{l}\geq 1 \text { dose } P C V_{13} \text {, excluding children with any medical } \\
\text { condition }{ }^{c}\end{array}$ & $16 / 1$ & $117 / 28$ & $\begin{array}{c}4.41 \\
(0.52-37.18) \\
\end{array}$ & 0.173 & $\begin{array}{c}5 \cdot 51 \\
(0.64-47.19)\end{array}$ & 0.120 \\
\hline
\end{tabular}

$\mathrm{Cl}$ : confidence interval; Mix: schedules consisting of PCV13 and another PCV; OR: matched odds ratio with unvaccinated children as the reference group; PCV: pneumococcal conjugate vaccine; Unvac: unvaccinated (children with zero doses of any PCV); Vac: vaccinated.

${ }^{a}$ Conditional logistic regression.

${ }^{b}$ Conditional logistic regression adjusted for sex and parental income level.

c Any medical condition was defined as major chronic illness (cardiovascular, respiratory, neurological, renal or hepatic disease, diabetes, immunosuppression or cancer), premature birth ( $<37$ weeks' gestation), or low birthweight ( $(2,500 \mathrm{~g})$. 
reference group (HR: $2.84 ; 95 \% \mathrm{Cl}: 1.02-7.88$ ) (Table 2).

Taking the period of $\mathrm{PCV}_{7}$ use (2005-2009) as the reference, we evaluated the effect of PCV13 in 2011-2014, obtaining an estimate of the indirect protective effect in unvaccinated children of $70 \%$ (HR: $0.30 ; 95 \% \mathrm{Cl}$ : $0.12-0.77)$ and a total effect in children with $\mathrm{PCV}_{13}$ of 61\% (HR: 0.39; 95\% Cl: 0.22-0.70) against all-serotype IPD. In a similar analysis limited to IPD cases from PCV13 serotypes, the total effect was 90\% (HR: 0.10; 95\% Cl: 0.03-0.32) (Table 3).

\section{Evaluation of the direct vaccine effect}

Between July 2010 and December 2014, 34 cases of IPD were included in the case-control study. The median age was 18.9 months (range 3.4-57.7 months). Clinical presentations were bacteraemia (17 cases), pneumonia (14 cases) and meningitis (three cases). In 30 cases the serotype was available: 10 cases were caused by $\mathrm{PCV}_{13}$ serotypes and 20 cases by non-PCV13 serotypes. There were two vaccine failures due to serotype 3 in immunocompetent children who had received four doses of $P C V_{13}$. Additionally, there was one case due to serotype 19A (a PCV13-only serotype) in a child who had received three doses of $\mathrm{PCV}_{10}$ and a booster dose of $P C V_{13}$. All but one of the seven cases in unvaccinated children were due to $P \mathrm{CV}_{13}$ serotypes, while most of the cases in vaccinated children (18 of 21) were caused by non-PCV13 serotypes.

The 34 cases and 272 matched controls presented similar sociodemographic characteristics and underlying medical conditions, with the exception of a higher proportion of males in cases ( $71 \%$ vs $51 \%, p=0.037$ ) (Table 4).

Cases and controls were similar in PCV vaccination history: 27 cases (79\%) and 217 controls (80\%) had received at least one dose of any PCV, and 24 cases (71\%) and 198 controls (73\%) had received PCV13. There were no children with a single dose given o-14 days before the reference date. The adjusted direct effect of at least one dose of $\mathrm{PCV}_{13}$ (including schedules consisting of $\mathrm{PCV}_{13}$ and another $\mathrm{PCV}$ ) in preventing IPD caused by PCV13 serotypes was 95\% (OR: 0.05; $95 \% \mathrm{Cl}: 0.01-0.55 ; \mathrm{p}=0.014)$, and $93 \%(\mathrm{OR}: 0.07 ; 95 \%$ $\mathrm{Cl}: 0.01-0.70 ; p=0.023)$ when restricting the analysis to IPD due to additional PCV13 serotypes. Conversely, cases due to non-PCV13 serotypes had a higher odds of $\mathrm{PCV}_{13}$ vaccination than controls, although with a wide $\mathrm{Cl}$ including the null effect (OR: $6.75 ; 95 \% \mathrm{Cl}$ : $0.77-59.19 ; p=0.085)$. As a result, we did not detect a significant direct effect of $\mathrm{PCV}_{13}$ in preventing allserotype IPD (OR: 0.91; 95\% Cl: 0.33-2.49; $\mathrm{p}=0.848$ ). Similar findings were obtained for schedules of $\mathrm{PCV}_{13}$ only, when analysing the effect of at least three doses of $\mathrm{PCV}_{13}$, and when excluding children with any medical condition (Table 5).

\section{Discussion}

In a cohort of children younger than 5 years followed up during the 14 years in which $\mathrm{PCV}_{7}$ was introduced and its subsequent replacement by $P \mathrm{CV}_{13}$, we observed large reductions in the incidence of IPD, both in vaccinated children (total effect, $76 \%$ ) and in those not vaccinated (indirect effect, $78 \%$ ). The effect against PCV-serotype cases was earlier and more pronounced in vaccinated than in unvaccinated children, but these differences disappeared when we evaluated the effect against IPD due to all serotypes.

The replacement of $\mathrm{PCV}_{7}$ by $\mathrm{PCV}_{13}$ was followed by a reduction of $90 \%$ in the incidence of IPD due to $\mathrm{PCV}_{13}$ serotypes in children who had received PCV13.

PCV13 effectiveness (direct effect) was 95\% against IPD due to PCV13 serotypes.

Other studies have also reported a high effectiveness of $\mathrm{PCV}_{13}$ against IPD due to vaccine serotypes: $86 \%$ in Quebec, Canada [8], and 75\% in the UK [7], in the first 3 and 3.5 years after $\mathrm{PCV}_{13}$ introduction, respectively. In our case-control analysis, as in the study from Quebec, the effectiveness estimate for non-PCV13 serotypes was negative, although with a wide $\mathrm{Cl}$ including the null effect.

In our cohort analysis, the incidence of IPD due to nonPCV13 serotypes among children who had received $\mathrm{PCV}_{13}$ increased compared with the incidence in unvaccinated children in the pre-PCV period. This finding suggests some vaccine-induced replacement, a phenomenon well-documented for $\mathrm{PCV}_{7}[3,24-27]$, which may be beginning to occur with PCV13 $[12,19$ $21,28]$. Nevertheless, the initial incidence of non- $\mathrm{PCV}_{13}$ serotype IPD was low, and its increase has been much smaller than the reduction in vaccine serotype incidence, resulting in a considerable net population benefit of vaccination. The replacement effect may be a potential source of bias to be corrected for in indirect cohort studies $[6,7]$.

The two vaccine failures observed in children completely vaccinated with $\mathrm{PCV}_{13}$ were due to serotype 3 , for which different studies suggest lower effectiveness $[6,7,11,29]$.

To the best of our knowledge, ours is the first study to estimate the indirect effect of PCV13 against IPD in children younger than 5 years, and the results are consistent with those of other studies that have described reductions in IPD incidence in unvaccinated age groups after the change to $\mathrm{PCV}_{13}[10,11,17-19]$. A study in Boston in the United States between July 2010 and June 2012 observed an indirect effect against nasopharyngeal colonisation in unimmunised children as vaccine uptake reached $75 \%$ [30], however, we observed an indirect effect against $\mathrm{PCV}_{7}$ serotypes starting in the period $2005-2007$, when coverage reached only $48 \%$. The strong indirect effect of $\mathrm{PCV}_{13}$, added to serotype 
replacement in vaccinated children, leads to apparently paradoxical results, such as the low or absent direct effect of the vaccine against all-serotype IPD. In this situation the total effect is the measure that best reflects the benefit in the vaccinated population.

This study has certain limitations. Although the study size was small, it was enough to sustain the statistically significant findings presented in the results but not for more disaggregated analysis. Some of the estimates' Cls were wide and should be interpreted with caution. In the comparisons between periods in the cohort study, we cannot rule out the possibility that some of the changes detected could have been due to temporal fluctuations in specific serotype incidence unrelated to vaccine use, giving an over- or underestimation of the indirect and total vaccine effects. However, the high effectiveness of $\mathrm{PCV}_{13}$ against vaccine serotypes was confirmed in the case-control study limited to the last period, in an analysis not affected by temporal fluctuations. The cohort analysis took into account age, sex, period and PCV status, but was not adjusted for other variables. Although some residual confounding could be possible, the results were consistent with those of the case-control analysis in which we did adjust for other variables. The fact that the same study has found a protective effect of PCVs for vaccine serotypes but not for non-vaccine serotypes also argues against important residual confounding. $\mathrm{PCV}_{7}$ was already available in the baseline period (2001-2004), with an average coverage of $11 \%$; accordingly, some indirect effect cannot be ruled out. Nonetheless, the incidence of IPD in children younger than 5 years was still very high (75 per 100,000 PY), indicating little impact of vaccination. Few cases were not serotyped, and they were excluded from some analyses; however, sensitivity analyses were performed in the cohort study assigning these cases alternately to each serotype group (data not shown), and the main results were hardly affected. Only the indirect effect against $\mathrm{PCV}_{7}$ serotypes in the 2005-2007 period and the increased risk of nonPCV13 serotype IPD among vaccinated children in the 2011-2014 period lost statistical significance ( $p$ values 0.063 and 0.084 , respectively).

This study also has a number of strengths. Populationbased surveillance was active and consistent throughout the follow-up period. The case-control design achieved good comparability by individual matching, and was also adjusted for relevant covariables. The intermediate levels of vaccine coverage allowed a sufficient number of vaccinated and unvaccinated individuals to evaluate the direct and indirect effects.

In conclusion, $\mathrm{PCV}_{13}$ was highly effective in preventing vaccine-serotype IPD. With vaccine coverage around $76 \%$ in children, PCV benefits have been substantial and similar in vaccinated and unvaccinated children through strong total and indirect effects. Signs of possible serotype replacement in vaccinated children highlight the importance of ongoing surveillance and development of new pneumococcal vaccines. Joint assessment of vaccine effects at the individual and population level helps to better understand the complex dynamics of changes in the epidemiology of IPD that follow changes in the pneumococcal vaccination programme. The important vaccine benefit at the population level supports the recommendation for universal $P C V$ vaccination in children.

Members of the Working Group for Surveillance of the Pneumococcal Disease in Navarra

Judith Chamorro, Mercedes Gabari, Pilar Artajo, Luz Peña, Nuria Clerigué, Isabel Polo, Carmen Martín, Ana Mazón, Laura Moreno-Galarraga, Natividad Viguria-Sánchez (Complejo Hospitalario de Navarra); José Leiva, Jorge $M$. Núnez-Córdoba (Clínica Universidad de Navarra); María Teresa Ortega, Montse Torres, José Javier García-Irure (Hospital Reina Sofía de Tudela); Laura Barrado, Francisco Lameiro (Hospital García Orcoyen de Estella); Nerea Álvarez (Servicio Navarro de Salud); Manuel García Cenoz, Fátima Irisarri, Maite Arriazu, Itziar Casado, Iván Martínez-Baz, Agurtzane Zabala and Jesús Castilla (Instituto de Salud Pública de Navarra).

\section{Acknowledgements}

This work was partially supported by SpIDnet (Assessing the impact of vaccination with the conjugate vaccines on the epidemiology of invasive pneumococcal disease in Europe), a network funded by the European Centre for Disease Prevention and Control (ECDC/2012/038). We thank Dr Asunción Fenoll at the National Centre of Microbiology (Instituto de Salud Carlos III, Majadahonda, Spain) for serotyping of the pneumococcal isolates.

\section{Conflict of interest}

Enrique Bernaola has collaborated as a researcher in a clinical trial of $\mathrm{PCV}_{13}$ for Pfizer Inc. All other authors declare no competing interests.

\section{Authors' contributions}

$M G, A B$ and $J C$ designed the study. MG and JC performed the statistical analyses and drafted the manuscript. $\mathrm{MH}, \mathrm{FG}$ and EB participated in the data review and manuscript revision. $\mathrm{LT}, \mathrm{AG}$ and CE were responsible for the microbiological results and participated in the manuscript revision. Members of the Working Group for Surveillance of the Pneumococcal Invasive Disease in Navarra participated in the data collection and data review. All authors contributed to data interpretation, revised the article critically and approved the final version.

\section{References}

1. Whitney CG, Pilishvili T, Farley MM, Schaffner W, Craig AS Lynfield R, et al. Effectiveness of seven-valent pneumococcal conjugate vaccine against invasive pneumococcal disease: a matched case-control study. Lancet. 2006;368(9546):1495502. DOI: 10.1016/S0140-6736(06)69637-2 PMID: 17071283

2. Lucero MG, Dulalia VE, Nillos LT, Williams G, Parreño RA, Nohynek $\mathrm{H}$, et al. Pneumococcal conjugate vaccines for preventing vaccine-type invasive pneumococcal disease and X-ray defined pneumonia in children less than two years of age. Cochrane Database Syst Rev. 2009;7(4):CDoo4977.DOI: 10.1002/14651858.CD 004977.pub2 PMID:19821336 PMID: 19821336 
3. Weinberger DM, Malley R, Lipsitch M. Serotype replacement in disease after pneumococcal vaccination.Lancet. 2011;378(9807):1962-73. DOI: 10.1016/S0140-6736(10)62225-8 PMID: 21492929

4. Rozenbaum MH, Boersma C, Postma MJ, Hak E. Observed differences in invasive pneumococcal disease epidemiology after routine infant vaccination. Expert Rev Vaccines. 2011;10(2):187-99. DOI: 10.1586/erv.10.163 PMID: 21332268

5. World Health Organization (WHO). Recommendations for the production and control of pneumococcal conjugate vaccines. Technical Report Series 927, fifty-fourth report. Annex 2. Geneva: WHO; 2005. Available from: http://apps.who.int/ medicinedocs/documents/s16102e/s16102e.pdf

6. Miller E, Andrews NJ, Waight PA, Slack MP, George RC. Effectiveness of the new serotypes in the 13-valent pneumococcal conjugate vaccine.Vaccine. 2011;29(49):9127-31. DOI: 10.1016/j.vaccine.2011.09.112 PMID: 21983361

7. Andrews NJ, Waight PA, Burbidge P, Pearce E, Roalfe L, Zancolli $M$, et al. Serotype-specific effectiveness and correlates of protection for the 13 -valent pneumococcal conjugate vaccine: a postlicensure indirect cohort study. Lancet Infect Dis. 2014;14(9):839-46. DOI: 10.1016/S1473-3099(14)70822-9 PMID: 25042756

8. Deceuninck G, De Serres G, Boulianne N, Lefebvre B, De Wals P. Effectiveness of three pneumococcal conjugate vaccines to prevent invasive pneumococcal disease in Quebec, Canada.Vaccine. 2015;33(23):2684-9. DOI: 10.1016/j. vaccine.2015.04.005 PMID: 25887086

9. Davis SM, Deloria-Knoll M, Kassa HT, O’Brien KL. Impact of pneumococcal conjugate vaccines on nasopharyngeal carriage and invasive disease among unvaccinated people: review of evidence on indirect effects.Vaccine. 2013;32(1):133-45. DOI: 10.1016/j.vaccine.2013.05.005 PMID: 23684824

10. Harboe ZB, Dalby T, Weinberger DM, Benfield T, Mølbak K Slotved HC, et al. Impact of 13-valent pneumococcal conjugate vaccination in invasive pneumococcal disease incidence and mortality. Clin Infect Dis. 2014;59(8):1066-73. DOI: 10.1093/ cid/ciu524 PMID: 25034421

11. Moore MR, Link-Gelles R, Schaffner W, Lynfield R, Lexau C, Bennett NM, et al. Effect of use of 13 -valent pneumococcal conjugate vaccine in children on invasive pneumococcal disease in children and adults in the USA: analysis of multisite, population-based surveillance. Lancet Infect Dis. 2015;15(3):301-9. DOI: 10.1016/S1473-3099(14)71081-3 PMID: 25656600

12. Waight PA, Andrews NJ, Ladhani SN, Sheppard CL, Slack MP, Miller E. Effect of the 13-valent pneumococcal conjugate vaccine on invasive pneumococcal disease in England and Wales 4 years after its introduction: an observational cohort study. Lancet Infect Dis. 2015;15(5):535-43. DOI: 10.1016/S14733099(15)70044-7 PMID: 25801458

13. Halloran ME, Struchiner CI, Longini IM. Study designs for evaluating different efficacy and effectiveness aspects of vaccines.Am J Epidemiol. 1997;146(10):789-803. DOI: 10.1093/ oxfordjournals.aje.aoo9196 PMID: 9384199

14. Hanquet G, Valenciano M, Simondon F, Moren A. Vaccine effects and impact of vaccination programmes in postlicensure studies.Vaccine. 2013;31(48):5634-42. DOI: 10.1016/j. vaccine.2013.07.006 PMID: 23856332

15. Instituto de Salud Pública de Navarra [Public Health Institute of Navarra]. Instrucciones sobre la vacunación de adultos y en situaciones clínicas especiales [Instructions on vaccination of adults and patients in special clinical situations]. Boletín Informativo. 2013;72:1-3. Spanish. [Accessed July 2015]. Available from: http://www.navarra.es/NR/rdonlyres/ AECCD760-AB2A-4841-818A-FA53478FD6DC/255921/BOL72INT. pdf

16. Advisory Committee on Vaccines of the Spanish Association of Pediatrics, Moreno-Pérez D, Alvarez García FJ, Aristegui Fernández J, Barrio Corrales F, Cilleruelo Ortega MJ, Corretger Rauet JM, et al. . Immunization schedule of the Spanish Association of Pediatrics: 2012 recommendations. An Pediatr (Barc). 2012;76(1):43.e1-23. DOI: 10.1016/j.anpedi.2011.10.008 PMID: 22177960

17. Working Group for Surveillance of the Pneumococcal Disease in Navarre,Guevara M, Ezpeleta C, Gil-Setas A, Torroba L, Beristain X, Aguinaga A, et al. . Reduced incidence of invasive pneumococcal disease after introduction of the 13-valent conjugate vaccine in Navarre, Spain, 2001-2013.Vaccine. 2014;32(22):2553-62. DOI: 10.1016/j.vaccine.2014.03.054 PMID: 24674661

18. Oxford Invasive Pneumococcal Surveillance Group,Moore CE, Paul J, Foster D, Mahar SA, Griffiths D, Knox K, et al. . Reduction of invasive pneumococcal disease 3 years after the introduction of the 13 -valent conjugate vaccine in the Oxfordshire region of England.J Infect Dis. 2014;210(7):1001-11. DOI: 10.1093/infdis/jiu213 PMID: 24719477
19. Steens A, Bergsaker MA, Aaberge IS, Rønning K, Vestrheim DF. Prompt effect of replacing the 7-valent pneumococcal conjugate vaccine with the 13-valent vaccine on the epidemiology of invasive pneumococcal disease in Norway.Vaccine. 2013;31(52):6232-8. DOI: 10.1016/j. vaccine.2013.10.032 PMID: 24176490

20. Portuguese Group for the Study of Streptococcal Infections, Aguiar SI, Brito MJ, Horacio AN, Lopes JP, Ramirez $M$, Melo-Cristino l, et al. . Decreasing incidence and changes in serotype distribution of invasive pneumococcal disease in persons aged under 18 years since introduction of 10 -valent and 13 -valent conjugate vaccines in Portugal, July 2008 to June 2012. Euro Surveill. 2014;19(12):20750. DOI: 10.2807/1560-7917. ES2014.19.12.20750 PMID: 24698140

21. Microbiologists of Epibac,Lepoutre A, Varon E, Georges $S$, Dorléans F, Janoir C, Gutmann L, et al. Impact of the pneumococcal conjugate vaccines on invasive pneumococcal disease in France, 2001-2012.Vaccine. 2015;33(2):359-66. DOI: 10.1016/j.vaccine.2014.11.011 PMID: 25448105

22. Instituto Nacional de Estadística, España [National Statistics Institute, Spain]. INEbase. Estadística del Padrón Continuo. Datos nacionales, por CCAA y por provincias. [Continuous Register Statistics. National level data, by autonomous community and province] [Accessed July 2015]. Available from: http://www.ine.es/jaxi/menu.do?type=pcaxis \& path=/t2o/ e245/\&file=inebase.

23. Aguilar I, Reyes M, Martínez-Baz I, Guevara M, Albeniz E, Belza $M$, et al. Use of the vaccination register to evaluate influenza vaccine coverage in seniors in the 2010/11 influenza season, Navarre, Spain. Euro Surveill. 2012;17(17):20154.PMID: 22551499

24. Singleton RJ, Hennessy TW, Bulkow LR, Hammitt LL, Zulz T, Hurlburt DA, et al. Invasive pneumococcal disease caused by nonvaccine serotypes among alaska native children with high levels of 7-valent pneumococcal conjugate vaccine coverage. JAMA. 2007;297(16):1784-92. DOI: 10.1001/jama.297.16.1784 PMID: 17456820

25. Guevara M, Barricarte A, Gil-Setas A, García-Irure JJ, Beristain $X$, Torroba $L$, et al. Changing epidemiology of invasive pneumococcal disease following increased coverage with the heptavalent conjugate vaccine in Navarre, Spain. Clin Microbiol Infect. 2009;15(11):1013-9. DOI: 10.1111/j.14690691.2009.02904.x PMID: 19673968

26. van Gils EJ, Veenhoven RH, Hak E, Rodenburg GD, Keijzers WC, Bogaert D, et al. Pneumococcal conjugate vaccination and nasopharyngeal acquisition of pneumococcal serotype 19A strains. JAMA. 2010;304(10):1099-106. DOI: 10.1001/ jama.2010.1290 PMID: 20823436

27. Serotype Replacement Study Group,Feikin DR, Kagucia EW, Loo JD, Link-Gelles R, Puhan MA, Cherian T, et al. . Serotype-specific changes in invasive pneumococca disease after pneumococcal conjugate vaccine introduction: a pooled analysis of multiple surveillance sites.PLoS Med. 2013;10(9):e1001517. DOI: 10.1371/journal.pmed.1001517 PMID: 24086113

28. van der Linden M, Perniciaro S, Imöhl M. Increase of serotypes $15 A$ and $23 B$ in IPD in Germany in the $P C V_{13}$ vaccination era. BMC Infect Dis. 2015;15(1):207. DOI: 10.1186/s12879-015-09419 PMID: 25940580

29. Nunes MC, Madhi SA. Review on the immunogenicity and safety of PCV-13 in infants and toddlers. Expert Rev Vaccines. 2011;10(7):951-80. DOI: 10.1586/erv.11.76 PMID: 21806394

30. Loughlin AM, Hsu K, Silverio AL, Marchant CD, Pelton SI. Direct and indirect effects of $\mathrm{PCV}_{13}$ on nasopharyngeal carriage of PCV13 unique pneumococcal serotypes in Massachusetts' children.Pediatr Infect Dis J. 2014;33(5):504-10. DOI: 10.1097/ INF.0000000000000279 PMID: 24670957

\section{License and copyright}

This is an open-access article distributed under the terms of the Creative Commons Attribution (CC BY 4.0) Licence. You may share and adapt the material, but must give appropriate credit to the source, provide a link to the licence, and indicate if changes were made.

This article is copyright of the authors, 2016. 